\title{
Primeiro isolamento ambiental de Cryptococcus gattii no Estado do Espírito Santo
}

\author{
First isolation of Cryptococcus gattii from the environment \\ in the State of Espírito Santo
}

Ludmila de Matos Baltazare Mariceli Araújo Ribeiro ${ }^{1}$

\begin{abstract}
RESUMO
A presença de Cryptococcus gattii foi investigada em diferentes regiões do Estado do Espírito Santo. A maioria (73) das amostras foi coletada de árvores localizadas em lugares públicos de Vitória; 47 amostras foram coletadas de áreas preservadas ou ainda com pouco impacto humano, situados nos arredores desta cidade, a altitudes entre 0 e $900 \mathrm{~m}$ acima do nível do mar e 48 de árvores nativas das regiões norte e sul do estado. As amostras foram coletadas de ocos e troncos de árvores com auxílio de swab e resultaram em $2(1,2 \%)$ isolados de Cryptococcus neoformans, 2 (1,2\%) de Cryptococcus gattii e $1(0,6 \%)$ de Cryptococcus laurentii. A espécie Cryptococcus gattii foi encontrada somente em árvores nativas da região norte, áreas que ainda apresentam resquícios de Floresta Atlântica, enquanto todas as amostras obtidas de vinte e duas espécies de árvores localizadas em área urbana não permitiram a detecção de Cryptococcus gattii. Esses resultados mostram uma possível relação entre ocorrência de Floresta Atlântica e Cryptococcus gattii e confirma que o meio ambiente é fonte de infecção desse fungo.
\end{abstract}

Palavras-chaves: Cryptococcus gattii. Meio ambiente. Árvores. Espírito Santo.

\section{ABSTRACT}

The presence of Cryptococcus gattii was investigated in different regions of the State of Espírito Santo. The largest number (73) of samples was collected from trees located in public places in Vitória; 47 came from preserved areas or areas with only minor human impact, surrounding this city, at altitudes from 0 to $900 \mathrm{~m}$ above sea level; 48 came from trees native of the northern and southern regions of the state. The samples were collected from tree hollows and trunks by of swabs and yielded two isolates (1.2\%) of Cryptococcus neoformans, two (1.2\%) of Cryptococcus gattii and one (0.6\%) of Cryptococcus laurentii. The species Cryptococcus gattii was found only in native trees from the northern region, in areas that still have remains of the Atlantic Forest, while none of the samples from any of the 22 tree species located in urban areas was able to show the presence of Cryptococcus gattii. These results show a possible relationship between the presence of Atlantic Forest and occurrences of Cryptococcus gattii. They confirm that the environment is a source of infection with this fungus.

Key-words: Cryptococcus gattii. Environment. Trees. Espírito Santo.

O fungo basidiomiceto Cryptococcus apresenta duas espécies consideradas patogênicas para seres humanos e animais ${ }^{150}$, Cryptococcus gattii e Cryptococcus neoformans, agentes etiológicos da criptococose, micose que afeta tanto indivíduos saudáveis ${ }^{25}$, como imunocomprometidos ${ }^{625}$, especialmente pacientes com $\operatorname{AIDS}^{425}$. A infecção é adquirida através da inalação de propágulos do fungo: leveduras desidratadas ou basidiósporos ${ }^{11}{ }^{20}$ e após a inalação pode haver disseminação hematogênica ${ }^{25}$ para tecido cutâneo, órgãos internos e/ou sistema nervoso central, onde se observa a forma clínica mais comum da micose, a meningoencefalite ${ }^{25}$.
Ambas as espécies Cryptococcus gattii e Cryptococcus neoformans são capazes de produzir lacase, que permite a colonização da madeira, principalmente em avançado estado de decomposição ${ }^{1221}$. Sua distribuição geográfica por muito tempo foi considerada restrita a regiões tropicais e subtropicais ${ }^{4112433}$. Entretanto, esse paradigma, atualmente, foi quebrado, uma vez que foi relatada a ocorrência de Cryptococcus gattii em regiões de clima temperado ${ }^{514273840}$. Cryptococcus neofomans por sua vez, ocorre geralmente em excrementos secos principalmente de pombos $^{1131}$.

1. Núcleo de Doenças Infecciosas, Universidade Federal do Espírito Santo, Vitória, ES.

Apoio financeiro: Fundação de Apoio a Ciência e Tecnologia do Estado do Espírito Santo (FAPES), processo nº 30806665/2005.

Endereço para correspondência: Dra. Mariceli Araujo Ribeiro. Núcleo de Doenças Infecciosas/UFES. Av. Marechal Campos $n^{\circ}$ 1468, Maruípe, $29040-093$ Vitória, ES. Telefax: 5527 3335-7498

e-mail: mariceliaraujo@yahoo.com

Recebido para publicação em 05/05/2008

Aceito em 01/09/2008 
O objetivo deste estudo foi investigar a ocorrência de Cryptococcus gattii em áreas da grande Vitória e em diferentes regiões do Estado do Espírito Santo.

\section{MATERIAL E MÉTODOS}

Coleta das amostras. No período de julho de 2005 a novembro de 2006, foram coletadas 168 amostras de oco e de casca de árvores. Os locais foram selecionados com base na observação de fatores de risco para a aquisição de Cryptococcus gattii pela população em geral: locais urbanos de Vitória com árvores ornamentais, além de regiões localizadas no Sul e no Norte do estado, com diferentes tipos de vegetação.

Colheita e processamento das amostras. As amostras foram obtidas com auxílio de swab, baseada na metodologia proposta por Granados \& Castañeda ${ }^{11}$. No momento da colheita 0 swab, foi passado na superfície e nos ocos das árvores e transferido para tubos, previamente identificados, contendo meio de transporte de Stuart (Difco). No laboratório, cada swab foi passado na superfície de 10 placas de ágar com semente de Níger ${ }^{11}{ }^{37}$ (Guizotia abyssinica), que foram incubadas por até cinco dias a $35^{\circ} \mathrm{C}$.

Isolamento e identificação das leveduras em nível de gênero e espécie. Todas as colônias de coloração marrom no ágar Níger foram repicadas para ágar sabouraud dextrose (Difco). A identificação do gênero Cryptococcus foi baseada na detecção da enzima urease em Meio de Christensen (Difco), na ausência de fermentação de carboidratos e na assimilação obrigatória de inositol como única fonte de carbono. Para caracterização das espécies Cryptococcus neoformans e Cryptococcus gattii foi analisado o perfil de assimilação de carboidratos, a ausência de assimilação de nitrato como fonte de nitrogênio inorgânico e características micromorfológicas típicas, como a visualização de cápsula em preparação microscópica com tinta Nankin. As espécies Cryptococcus neoformans e Cryptococcus gattii foram diferenciadas no meio CGB (Canavanina-Glicina-Azul de bromotimol) ${ }^{1136}$.

\section{RESULTADOS}

Do total de 168 amostras de árvores, 5 (3\%) foram positivas para Cryptococcus spp, sendo 2 (1.2\%) positivas para Cryptococcus neoformans, 2 (1.2\%) para Cryptococcus gattii e uma (0.6\%) para Cryptococcus laurentii. As amostras foram coletadas em oito áreas dentro da região metropolitana de Vitória, incluindo parques, horto e campi da UFES (73 amostras). Foram também incluídas duas áreas de preservação ambiental (15 amostras) localizadas respectivamente a 27 e $62 \mathrm{~km}$ de Vitória: Reserva Biológica de Duas Bocas e Parque Estadual Paulo César Vinha, caracterizado por vegetação de restinga. Foram ainda selecionadas quatro localidades na região serrana (32 amostras), cerca de $80 \mathrm{~km}$ de Vitória, além de três na região norte (32 amostras) e duas na região sul do estado (16 amostras). As duas cepas de Cryptococcus gattii foram isoladas apenas de árvores da região norte do estado, incluindo a reserva biológica de Sooretama (Tabela 1 e Figura 1).

\begin{tabular}{|c|c|c|c|c|}
\hline Local & $\begin{array}{c}\text { Amostras } \\
\text { positivas (\%) }\end{array}$ & $\begin{array}{c}\text { Total } \\
\text { de amostras }\end{array}$ & Tipo de material vegetal & Referências \\
\hline \multicolumn{5}{|l|}{ Norte } \\
\hline Amazônia & - & - & oco de Guettarda acreana & Fortes e cols ${ }^{10}$ \\
\hline Piauí & $9(28,0 \%)$ & 32 & Moquilea tomentosa e duas da espécie Cassia grandis & Lazera e cols ${ }^{21}$ \\
\hline \multicolumn{5}{|l|}{ Sudeste } \\
\hline Espírito Santo & $0(0,0 \%)$ & 136 & $\begin{array}{l}\text { Mangifera indica (mangueira), Cassia fistula (cássia- } \\
\text { imperial), Licania tomentosa (oiti), Caesalpinia ferrea } \\
\text { (pau ferro), Senna siamea (cássia amarela), Cassia } \\
\text { ferruginea (canafístula), Tabebuia rosea (ipê rosa), } \\
\text { Tabebuia impetiginosa (ipê roxo), Pinus sp (pinheiro), } \\
\text { Baubinia variegata (unha-de-vaca), Persea gratissima } \\
\text { (abacaterio), Caesalpinia peltophoroides (sibipiruna), } \\
\text { Cestrum nocturnum (dama-da-noite), Chorisia speciosa } \\
\text { (paineira rosa), Caesalpinia echinata (pau brasil), } \\
\text { Tibouchina granulosa (quaresmeira), Piptadenia } \\
\text { colubrina (angico branco), Delonix regia (flamboyant), } \\
\text { Ficus microcarpa (figueira), Tamarindus indica } \\
\text { (tamarindo), Carica papaya (pé-de-mamão), Spondias } \\
\text { mombin (pé-de-cajá), troncos de árvores em } \\
\text { decomposição, árvores de Ecossistemas Costeiros } \\
\text { (Floresta de Restinga) e árvores de grande e médio } \\
\text { porte da Floresta Atlântica }\end{array}$ & $\begin{array}{l}\text { Presente estudo } \\
\text { (Baltazar e Ribeiro) }\end{array}$ \\
\hline Espírito Santo & $2(6,2 \%)$ & 32 & Árvores nativas de Mata Atlântica & $\begin{array}{l}\text { Presente estudo } \\
\text { (Baltazar e Ribeiro) }\end{array}$ \\
\hline São Paulo & $2(8,3 \%)$ & 24 & detritos de Eucalyptus spp & Montenegro e cols ${ }^{27}$ \\
\hline \multicolumn{5}{|l|}{ Sul } \\
\hline Rio Grande de Sul & $13(34,0 \%)$ & 38 & material fecal de pássaros & Abegg e cols ${ }^{1}$ \\
\hline
\end{tabular}




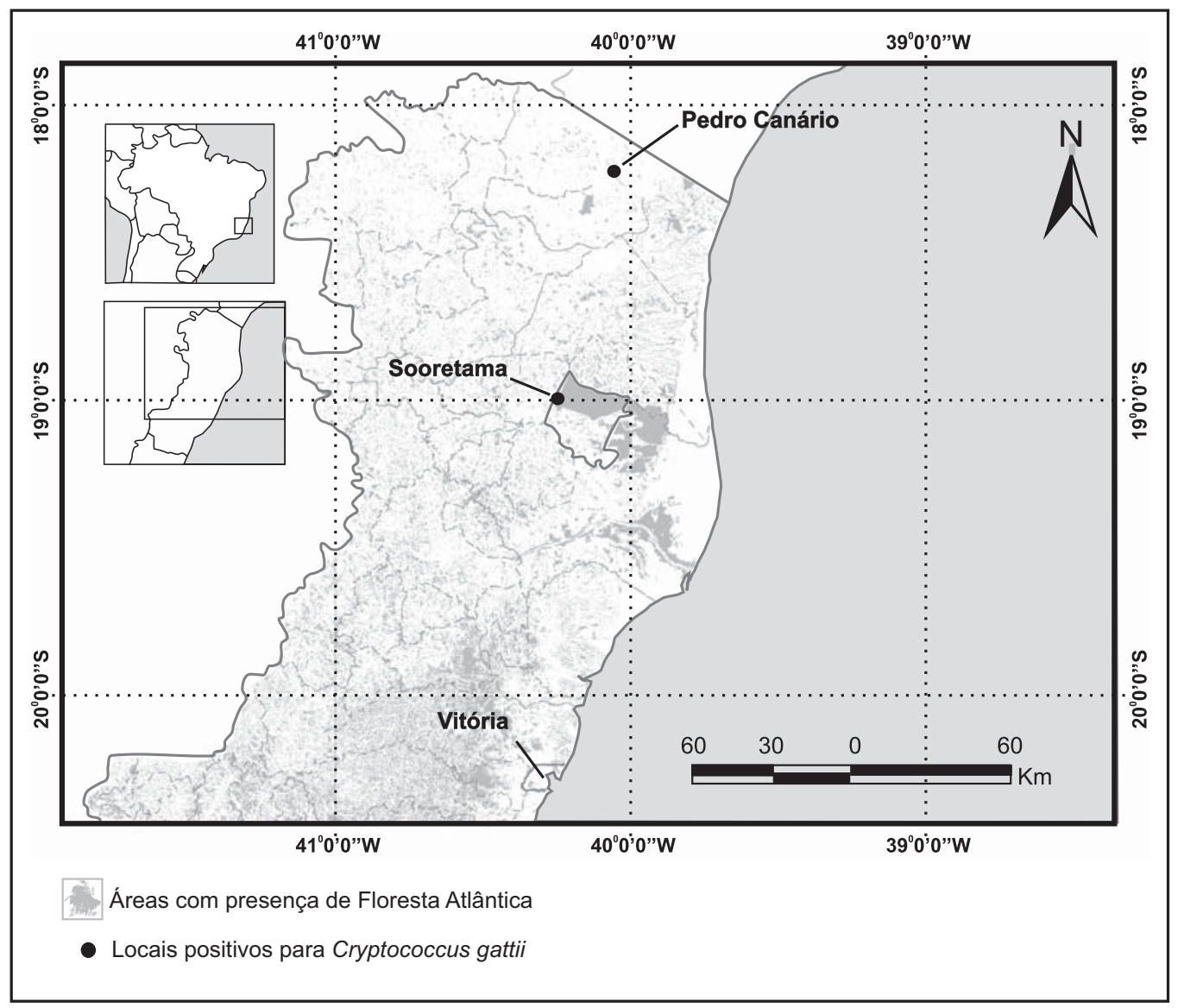

Figura 1 - Regiões do Estado do Espírito Santo de onde foram isoladas as cepas Cryptococcus gattii.

\section{DISCUSSÃo}

Por muito tempo a espécie Cryptococcus gattii foi estritamente associada a árvores de eucalipto (Eucalyptus camaldulensis) ${ }^{8} \mathrm{e}$ a climas tropicais e subtropicais ${ }^{27333940}$. Atualmente, entretanto, esta espécie tem sido encontrada em diferentes árvores e regiões geográficas (Tabela 2).

No Brasil, a espécie foi encontrada nas regiões Norte, Sul e Sudeste ${ }^{102127}$, embora sua maior frequiência seja na região Norte $^{27}$. Além de árvores, outros nichos de Cryptococcus gattii no ambiente também estão sendo detectados, como o achado desta espécie em excrementos de pássaros psitacídeos de cativeiro, em zoológico do Rio Grande do Sull (Tabela 1).

Neste estudo, um total de 22 espécies de árvores ornamentais e frutíferas foram identificadas, mas o isolamento do fungo não ocorreu nestes vegetais, indicando, como salienta Randhawa e $\operatorname{col}^{28}$, que a espécie pode ter distribuição muito generalizada, 0 que dificulta sua detecção na natureza. A espécie Cryptococcus gattii foi isolada de árvores existentes apenas nas regiões de Floresta Atlântica e, que por não apresentarem nomes populares nem estruturas como flores e frutos (informações imprescindíveis para a identificação de gimnospermas e angiospermas) não foi possível estabelecer as espécies destas árvores. Além disto, a cepa isolada em Pedro Canário foi coletada de uma árvore em adiantado estado de decomposição. Nenhuma espécie de Cryptococcus foi isolada de eucaliptos (mencionada anteriormente na literatura como a principal fonte de Cryptococcus gatti $i^{2729}$ ), coincidindo com achados no Canadá ${ }^{14}$ e outras regiões ${ }^{311} 1214162130$, e demonstrando que o eucalipto não é o nicho ecológico único e primário dessa espécie ${ }^{142140}$.

A baixa ocorrência de Cryptococcus gattii no Espírito Santo confirma os dados de outros estudos, onde a espécie tem sido isolada do meio ambiente em porcentagem menor que Cryptococcus neoformans ${ }^{23}{ }^{28}$. Nas regiões sul, sudeste e centro-oeste o isolamento de Cryptococcus gattii do ambiente é raro quando comparado a Cryptococcus neoformans (90,2\% e $1,9 \%$, respectivamente $)^{28}$. No entanto, esta espécie tem sido mais prevalente nas regiões norte e nordeste $(62 \%)^{28}$. Observa-se que mais de $50 \%$ das amostras foram coletadas de árvores presentes na região metropolitana de Vitória e em nenhuma delas foi encontrado Cryptococcus gattii, evidenciando que as condições climáticas de Vitória, cidade litorânea e com temperaturas médias elevadas, não são propícias para a ocorrência do fungo. Da mesma forma, Cryptococcus gattii não foi encontrado no Parque Estadual Paulo César Vinha, caracterizado por apresentar vegetação de restinga.

As amostras positivas para Cryptococcus gattii, foram procedentes de Sooretama e Pedro Canário, regiões localizadas ao norte do estado. Sooretama é uma região marcada pela presença de Floresta Atlântica, tanto próxima como afastada dos 
Tabela 2 - Isolamento ambiental de Cryptococcus gattii em diferentes regiões geográficas do mundo.

\begin{tabular}{|c|c|c|c|c|}
\hline País & $\begin{array}{c}\text { Amostras } \\
\text { positivas (\%) }\end{array}$ & $\begin{array}{l}\text { Total de } \\
\text { amostras }\end{array}$ & Tipo de material vegetal & Referências \\
\hline \multicolumn{5}{|l|}{ Oceania } \\
\hline Austrália & $3(30,0 \%)$ & 10 & Syncarpia glomulifera e outra árvore não identificada & Vilcins e cols ${ }^{40}$ \\
\hline \multicolumn{5}{|l|}{ Ásia } \\
\hline Índia & $5(1,3 \%)$ & 368 & $\begin{array}{l}\text { Eucalyptus camaldulensis, Eucalyptus citriodora e } \\
\text { Eucalyptus tereticornis }\end{array}$ & Chakrabarti e cols ${ }^{5}$ \\
\hline Índia & $7(10,6 \%)$ & 66 & Syzygium cumini e Ficus religiosa & Randhawa e cols ${ }^{32}$ \\
\hline Índia & $10(6,7 \%)$ & 148 & $\begin{array}{l}\text { oco de Mangifera indica, Tamarindus indica, } \\
\text { Pithecolobium dulce e tronco de Syzygium cumini }\end{array}$ & Grover e $\operatorname{cols}^{12}$ \\
\hline Jordânia & $0(0,0 \%)$ & 500 & - & Hamasha e cols ${ }^{13}$ \\
\hline Egito & $3(1,2 \%)$ & 245 & flor de Eucalyptus camaldulensis & Mahmoud e cols ${ }^{23}$ \\
\hline \multicolumn{5}{|l|}{ Europa } \\
\hline Itália & - & - & detritos de Eucalyptus camaldulensis & Montagna e $\operatorname{cols}^{26}$ \\
\hline \multicolumn{5}{|l|}{ América de Norte } \\
\hline Canadá & $58(0,8 \%)$ & 732 & $\begin{array}{l}\text { Alluns spp, Cedrus spp, Pseudotsuga spp e outras } \\
\text { não identificadas }\end{array}$ & Kidd e $\operatorname{cols}^{14}$ \\
\hline Canadá & $519(9,0 \%)$ & 5.704 & material de árvores, solo, ar, água potável, água do mar & Kidd e $\operatorname{cols}^{16}$ \\
\hline Estados Unidos & - & - & Eucalyptus camaldulensis & Pfeiffer e cols ${ }^{29}$ \\
\hline \multicolumn{5}{|l|}{ América Central } \\
\hline México & $7(28,0 \%)$ & 25 & detritos (tronco, folhas e solo) de Eucalyptus tereticornis & Licea e $\operatorname{cols}^{22}$ \\
\hline \multicolumn{5}{|l|}{ América do Sul } \\
\hline Colômbia & $2(3,0 \%)$ & 68 & detritos de Terminalia catappa & Callejas e cols ${ }^{3}$ \\
\hline Colômbia & - & - & tronco e amostras do solo de Eucalyptus camaldulensis & Gramados e cols ${ }^{11}$ \\
\hline Colômbia & $57(2,0 \%)$ & 2.816 & Eucalyptus spp, Laurus spp & Escandon e cols ${ }^{9}$ \\
\hline Argentina & $2(2,0 \%)$ & 100 & Eucalyptos spp & Davel e cols ${ }^{7}$ \\
\hline
\end{tabular}

centros populacionais. A Floresta Atlântica é caracterizada por ter árvores antigas, elevada umidade e sombreamento ${ }^{34}$ e parece ser um ambiente propício para a ocorrência de Cryptococcus gattii no Estado do Espírito Santo. Já Pedro Canário, é uma região caracterizada por desmatamentos recentes, mas que ainda resguarda resquícios de Floresta Atlântica. Possui também árvores em avançado estado de decomposição, condição que favorece 0 isolamento de Cryptococcus gattii ${ }^{2831}$.

Nossos resultados parecem demonstrar que Cryptococcus gattii preferencialmente ocorre em árvores nativas e localizadas em regiões ainda sem muita interferência humana, como sugerido por outros autores ${ }^{28}$.

Neste estudo, foi possível a detecção de três espécies de Cryptococcus: Cryptococcus gattii, Cryptococcus neoformans e Cryptococcus laurentii. Morfologicamente, as células das três espécies apresentaram cápsula, visualizadas ao microscópio óptico com tinta Nankin. É importante salientar a necessidade de se realizar testes de assimilação de carboidratos, além do emprego do meio CGB, pois Cryptococcus laurentii pode ter o mesmo padrão de crescimento de Cryptococcus gattii neste meio e deve ser diferenciado pela assimilação positiva da lactose ${ }^{20}$.

A detecção de Cryptococcus laurentii em material de árvore é um dado importante. Esta espécie tem sido encontrada em outros estados do Brasil ${ }^{17}{ }^{27}$ e pode ocasionalmente infectar também seres humanos ${ }^{18} 1935$.

Este estudo mostra a ocorrência de Cryptococcus gattii no norte do Espírito Santo e sua possível associação com microclima presente em Floresta Atlântica desta região. Outros estudos, no entanto, devem ser realizados para se estabelecer quais fatores podem estar diretamente relacionados à ocorrência do fungo no ambiente.

\section{AGRADECIMENTO}

Agradecemos a Claudiney Biral Santos, funcionário do Núcleo de Entomologia da Fundação Nacional de Saúde (FUNASA) no auxílio na colheita de amostras fora da grande Vitória.

\section{REFERÊNCIAS}

1. Abegg MA, Cella FL, Faganello J, Valente P, Schrank A, Vainstein MH. Cryptococcus neoformans and Cryptococcus gattii isolated from the excreta of psittaciformes in a southern Brazilian zoological garden. Mycopathologia 161:83-91, 2006.

2. Cafarchia C, Romito D, Iatta R, Camarda A, Montagna MT, Otranto D. Role of birds of prey as carriers and spreaders of Cryptococcus neoformans and other zoonotic yeasts. Medical Mycology 44:485-492, 2006.

3. Callejas A, Ordoñez N, Rodriguez MC, Castañeda E. First isolation of Cryptococcus neoformans var gattii, serotype $\mathrm{C}$, from the environment in Colombia. Medical Mycology 36: 341-344,1998.

4. Casali AK, Goulart L, Rosa e Silva LK, Ribeiro AM, Amaral AA, Alves SH, Schrank A, Meyer W, Vainstein MH. Molecular typing of clinical and environmental Cryptococcus neoformans isolates in the Brazilian state Rio Grande do Sul. Federation of European Microbiological Societies Yeast Research 3:405-415, 2003.

5. Chakrabarti A, Jatana M, Kumar P, Chatha L, Kaushal A, Padhye AA. Isolation of Cryptococcus neoformans var gattii from Eucalyptus camaldulensis in India. Journal of Clinical Microbiology 35:3340-3342, 1997.

6. Darzé C, Lucena R, Gomes I, Melo A. Características clínicas laboratoriais de 104 casos de meningoencefalite criptocócica. Revista da Sociedade Brasileira de Medicina Tropical 33: 21-26, 2000. 
7. Davel G, Abrantes R, Brudny M, Córdoba S, Rodero L, Canteros CE, Perrotta D. Primer aislamiento ambiental de Cryptococcus neoformans var gattii en Argentina. Revista Argentina de Microbiologia 35:110-112, 2003.

8. Ellis DH, Pfeiffer TJ. Natural habitat of Cryptococcus neoformans var gattii. Journal of Clinical Microbiology 28: 1642-1644, 1990.

9. Escandón P, Sánchez A, Martínez M, Meyer W, Castañeda E. Molecular epidemiology of clinical and environmental isolates of the Cryptococcus neoformans species complex reveals a high genetic diversity and the presence of the molecular type VGII mating type a in Colombia. Federation of European Microbiological Societies Yeast Research 6:625-635, 2006.

10. Fortes ST, Lazéra MS, Nishikawa MM, Macedo RC, Wanke B. First isolation of Cryptococcus neoformans var gattii from a native jungle tree in the Brazilian Amazon rainforest. Mycoses 44:137-140, 2001.

11. Granados DP, Castañeda E. Isolation and characterization of Cryptococcus neoformans varieties recovered from natural sources in Bogotá, Colombia, and study of ecological conditions in the area. Microbial Ecology 49:282-290, 2005.

12. Grover N, Nawange SR, Naidu J, Singh SM, Sharma A. Ecological niche of Cryptococcus neoformans var grubii and Cryptococcus gattii in decaying wood of trunk hollows of living trees in Jabalpur City of Central India. Mycopathologia 164:159-170, 2007.

13. Hamasha AM, Yildiran ST, Gonlum A, Saracli MA, Doganci L. Cryptococcus neoformans varieties from material under the canopies of eucalyptus trees and pigeon dropping samples from four major cities in Jordan. Mycopathologia 158:195-199, 2004.

14. Kidd SE, Chow Y, Mak S, Bach PJ, Chen H, Hingston AO, Kronstad JW, Bartlett KH. Characterization of environmental sources of the human and animal pathogen Cryptococcus gattii in British Columbia, Canada, and the Pacific Northwest of the United States. Applied and Environmental Microbiology 73:1433-1443, 2007.

15. Kidd SE, Guo H, Bartlett KH, Xu J, Kronstad JW. Comparative gene genealogies indicate that two clonal lineages of Cryptococcus gattii in British Columbia resemble strains from other geographical areas. Eukaryotic Cell 4:1629-1638, 2005.

16. Kidd SE, Hagen F, Tscharke RL, Huynh M, Bartlett KH, Fyfe M, Macdougall L, Boekhout T, Kwon-Chung KJ, Meyer W. A rare genotype of Cryptococcus gattii caused the cryptococcosis outbreak on Vancouver Island (British Columbia, Canada). Proceedings of the National Academy of Sciences of the Unidet States of American 101:17258-17263, 2004.

17. Kobayashi CC, Souza LK, Fernandes OF, Brito SC, Silva AC, Sousa ED, Silva MR. Characterization of Cryptococcus neoformans isolated from urban environmental sources in Goiânia, Goiás State, Brazil. Revista do Instituto de Medicina Tropical de São Paulo 47: 203-207, 2005.

18. Kordossis T, Avlami A, Velegraki A, Stefanou I, Georgakopoulos G, Papalambrou C, Legakis NJ. First report of Cryptococcus laurentii meningitis and a fatal case of Cryptococcus albidus cryptococcaemia in AIDS patients. Medical Mycology 36:335-339, 1998.

19. Kunova A, Krcmery V. Fungaemia due to thermophilic cryptococci: 3 cases of Cryptococcus laurentii bloodstream infections in cancer patients receiving antifungals. Scandinavian Journal of Infectious Diseases 31:328-331, 1999.

20. Kurtzman CP, Fell JW. The Yeast: A taxonomic study. Elsevier Science New York, 1998.

21. Lazera MS, Salmito Cavalcanti MA, Londero AT, Trilles L, Nishikawa MM, Wanke B. Possible primary ecological niche of Cryptococcus neoformans. Medical Mycology 38:379-383, 2000
22. Licea BA, Garza DG, Zuniga MT. Isolamento de Cryptococcus neoformans var gatti de Eucalytus tereticornis. Revista IberoAmericana Mycologia 13: 27-28, 1996.

23. Mahmoud YA. First environmental isolation of Cryptococcus neoformans var neoformans and var gattii from the Gharbia Governorate, Egypt. Mycopathologia 148: 83-86,1999.

24. Meyer W, Castañeda A, Jackson S, Huynh M, Castañeda E; IberoAmerican Cryptococcal Study Group. Molecular typing of IberoAmerican Cryptococcus neoformans isolates. Emerging Infectious Diseases 9:189-195, 2003.

25. Mitchell TG, Perfect JR. Cryptococcosis in the era of AIDS - 100 years after the discovery of Cryptococcus neoformans. Clinical Microbiology Reviews 8: 515-458, 1995

26. Montagna MT, Viviani MA, Pulito A, Aralla C, Tortorano AM, Fiore L, Barbuti S. Cryptococcus neoformans var gattii in Italy. Journal of Mycology Medical 7: 93-96, 1997

27. Montenegro H, Paula CR. Environmental isolation of Cryptococcus neoformans var gattii and Cryptococcus neoformans var. neoformans in the city of São Paulo, Brazil. Medical Mycology 38:385-390, 2000.

28. Nishikawa MM, Lazera MS, Barbosa GG, Trilles L, Balassiano BR, Macedo RC, Bezerra CC, Pérez MA, Cardarelli P, Wanke B. Serotyping of 467 Cryptococcus neoformans isolates from clinical and environmental sources in Brazil: analysis of host and regional patterns. Journal of Clinical Microbiology 41:73-77, 2003.

29. Pfeiffer T, Ellis D. Environmental isolation of Cryptococcus neoformans gattii from California. The Journal Infectious Diseases 163: 929-930, 1991.

30. Polacheck I, Platt Y, Aronovitch J. Catecholamines and virulence of Cryptococcus neoformans. Infection and Immunity 58:2919-2922, 1990.

31. Randhawa HS, Mussa AY, Khan ZU. Decaying wood in tree trunk hollows as a natural substrate for Cryptococcus neoformans and other yeast-like fungi of clinical interest. Mycopathologia 151:63-69, 2001.

32. Randhawa HS, Kowshik T, Khan ZU. Decayed wood of Syzygium cumini and Ficus religiosa living trees in Delhi/New Delhi metropolitan area as natural habitat of Cryptococcus neoformans. Medical Mycology 41:199-209, 2003.

33. Randhawa HS, Kowshik T, Preeti Sinha K, Chowdhary A, Khan ZU, Yan Z, Xu J, Kumar A. Distribution of Cryptococcus gattii and Cryptococcus neoformans in decayed trunk wood of Syzygium cumini trees in north-western India. Medical Mycology 44:623-630, 2006.

34. Raven PH, Evert RF, Eichhorn SE. Biologia Vegetal. Guanabara Koogan, Rio de Janeiro, 2001.

35. Ritterband DC, Seedor JA, Shah MK, Waheed S, Schorr I. A unique case of Cryptococcus laurentii keratitis spread by a rigid gas permeable contact lens in a patient with onychomycosis. Cornea 17:115-118, 1998.

36. Sorrell TC. Cryptococcus neoformans variety gattii. Medical Mycology 39: 155-168, 2001.

37. Staib F, Schultz-Dieterich J. Cryptococcus neoformans in fecal matter of birds kept in cages - control of Cryptococcus neoformans habitats. Zentralbi Bakteriology Mikrobiology Hygiene 179: 179-186, 1984

38. Stephen C, Lester S, Black W, Fyfe M, Raverty S. Multispecies outbreak of cryptococcosis on southern Vancouver Island, British Columbia. The Canadian Veterinary Journal 43: 792-794, 2002.

39. Tay ST, Lim HC, Tajuddin TH, Rohani MY, Hamimah H, Thong KL. Determination of molecular types and genetic heterogeneity of Cryptococcus neoformans and Cryptococcus gattii in Malaysia. Medical Mycology 44:617-622, 2006.

40. Vilcins I, Krockenberger M, Agus H, Carter D. Environmental sampling for Cryptococcus neoformans var. gattii from the Blue Mountains National Park Sydney, Australia. Medical Mycology 40:53-60, 2002. 